\title{
Analysis of Senescence-Related Differentiation Potentials and Gene Expression Profiles in Human Dental Pulp Stem Cells
}

\author{
Qiao Yia ${ }^{a-c}$ Ousheng Liu ${ }^{a, b, d}$ Fei Yan ${ }^{a-c}$ Xiao Lin ${ }^{c, e}$ Shu Diao ${ }^{c, f}$ \\ Liping Wang $^{c}$ Luyuan Jin ${ }^{c, f}$ Songlin Wang ${ }^{d, g}$ Yanqin Lu ${ }^{a, b} \quad$ Zhipeng Fan ${ }^{c}$ \\ ${ }^{a}$ Xiangya Stomatology Hospital and ${ }^{\mathrm{b}}$ School of Stomatology, Central South University, Changsha, and \\ 'Laboratory of Molecular Signaling and Stem Cell Therapy and ${ }^{\mathrm{d}}$ Molecular Laboratory for Gene Therapy and Tooth \\ Regeneration, Beijing Key Laboratory of Tooth Regeneration and Function Reconstruction, and Departments of \\ e Implant Dentistry, and f Pediatrics, Capital Medical University School of Stomatology, and 9Department of \\ Biochemistry and Molecular Biology, Capital Medical University School of Basic Medical Sciences, Beijing, China
}

\section{Key Words}

Dental pulp stem cells · Differentiation potentials .

Expression profile $\cdot$ Long noncoding RNA . Senescence

\begin{abstract}
Introduction: Dental pulp stem cell (DPSC)-mediated dental pulp regeneration is considered a promising method for the treatment of deep caries with pulpitis. However, mesenchymal stem cell (MSC) senescence is an adverse factor from the perspective of cell-based therapies. In this study, we investigated the characteristics and expression profiles of DPSCs from young and old donors. Methods: DPSCs from young and old donors were cultured in differentiation medium, and their differentiation potentials were assessed. Long noncoding RNA (LncRNA) microarray assays and a bioinformatic analysis were performed to investigate differences in LncRNA and mRNA expression profiles between DPSCs from young and old donors. Results: We found that DPSCs from young donors exhibited more powerful proliferation ability and greater osteogenic and adipogenic differentiation po-
\end{abstract}

Qiao Yi and Ousheng Liu contributed equally to this work.

\section{KARGER}

๑) 2016 S. Karger AG, Basel

E-Mail karger@karger.com

www.karger.com/cto tentials than DPSCs from old donors. In DPSCs from young donors, numerous LncRNAs were significantly up- $(n=389)$ or down-regulated $(n=172)$ compared to DPSCs from old donors. Furthermore, 304 mRNAs were differentially expressed, including 247 up-regulated genes and 57 downregulated genes in DPSCs from young donors. The bioinformatic analysis identified that several pathways may be associated with DPSC characteristics, such as those involved in the cell cycle and RNA transport, and revealed nuclear transcription factor $Y$ subunit $\beta$, general transcription factor IIB, and nuclear receptor subfamily 3 group $C$ member 1 as core regulatory factors and FR249114, FR299091, and ENST00000450004 as core LncRNAs. Conclusions: Our results indicated that senescence impaired the proliferation and differentiation potentials of DPSCs and that donor age is an important factor that affects their use for tooth regeneration. We also provide insight into the mechanisms responsible for senescence in DPSCs.

(c) 2016 S. Karger AG, Basel

Dr. Zhipeng Fan, Laboratory of Molecular Signaling and Stem Cell Therapy Beijing Key Laboratory of Tooth Regeneration and Function Reconstruction Capital Medical University School of Stomatology

No. 4, Tian Tan Xi Li, Beijing 100050 (China)

E-Mail zpfan@ccmu.edu.cn

Yanqin Lu

Xiangya Stomatology Hospital, Central South University

No. 72, Xiang Ya Road

Changsha 410078 (China)

E-Mail lu_yanqin@163.com 


\begin{tabular}{ll} 
Abbreviations used in this paper \\
\hline ALP & alkaline phosphatase \\
CEBPA & CCAAT/enhancer binding protein a \\
CENPK & centromere protein K \\
CFSE & Certified Functional Safety Expert \\
COL & collagen \\
COL1A2 & collagen type I a $_{2}$ \\
COL2 & collagen 2 \\
COL5 & collagen 5 \\
DPSC & dental pulp stem cell \\
FBXO5 & F-box protein 5 \\
GO & gene ontology \\
GPRC5A & G-protein-coupled receptor class C group 5 \\
& member A \\
GTF2B & general transcription factor IIB \\
GTF2F1 & general transcription factor IIF subunit 1 \\
IRF3 & interferon regulatory factor 3 \\
KEGG & Kyoto Encyclopedia of Genes and Genomes \\
LncRNA & long noncoding RNA \\
LPL & lipoprotein lipase \\
MSC & mesenchymal stem cell \\
NFY & nuclear transcription factor \\
NFYA & nuclear transcription factor Y subunit a \\
NFYB & nuclear transcription factor Y subunit $\beta$ \\
NR3C1 & nuclear receptor subfamily 3 group C member 1 \\
OPN & osteopontin \\
PCR & polymerase chain reaction \\
RUNX2 & runt-related transcription factor 2 \\
SOX9 & SRY (sex-determining region Y)-box 9 \\
TBP & TATA-box binding protein \\
TF & transcription factor \\
WNT & wingless-type MMTV integration site family \\
&
\end{tabular}

\section{Introduction}

Today, stem cell research is one of the most promising areas of biology because of its optimistic therapeutic implications. Stem cells derived from dental tissues, such as dental pulp, periodontal ligament, apical papilla, and dental follicle, are adult stem cells that could be used for regenerative medicine [Yen and Yelick, 2011]. Dental pulp stem cells (DPSCs), which have been recognized as mesenchymal stem cells (MSCs), are able to differentiate into various cell types, including osteoblasts, adipocytes, and chondrocytes. Many studies of tooth regeneration based on DPSCs have reported positive progress. For example, typical tooth-shaped tissues with balanced amelogenesis and dentinogenesis can be formed by DPSCs/apical bud cell recombinants in vivo [Yu et al., 2007]. In addition, pulp tissues, including those involved in the vasculature and innervation, and coronal dentin were previously regenerated after autologous transplantation of pulp stem cells with granulocyte-colony stimulating factor in a dog pulpectomized tooth [Iohara et al., 2013]. Moreover, a regenerated bioroot that exhibits characteristics of a normal tooth, including dentinal tubule-like and functional periodontal ligament-like structures, can be formed from a root-shaped hydroxyapatite tricalcium phosphate scaffold containing DPSCs and covered by a Vc-induced periodontal ligament stem cell sheet [Wei et al., 2013].

However, MSC aging is a negative process from the perspective of cell-based therapies, as all advantageous functions may become limited with age. There are clear losses in the proliferation capacity of MSCs with increasing donor age, including donor age-related decreases in the maximal life span and proliferation rate [Zaim et al., 2012]. The capacity of MSCs to differentiate into various tissue types changes with age, but the results of different studies are not consistent. Previous studies have indicated that MSCs isolated from different tissue sources are influenced differently by donor age. For instance, both the osteogenic and the adipogenic differentiation capacities of periodontal ligament stem cells decreased with increasing age [Zhang et al., 2012]. However, for adipose-derived stem cells, the osteogenic differentiation potential declined, and the adipogenic differentiation potential increased with age [Kornicka et al., 2015].

Many cellular and molecular processes change with advanced age, including epigenetic alterations, telomere attrition, loss of protein homeostasis, increased cellular senescence, stem cell exhaustion, and altered intercellular communication [López-Otín et al., 2013]. Clues regarding the molecules controlling MSC biology can be obtained by comparing MSCs with different biological activities. Emerging evidence suggests that long noncoding RNAs (LncRNAs) play important roles in a wide range of these molecular processes, which are the hallmarks of aging [Grammatikakis et al., 2014]. For example, LncRNAs can regulate the expression of some specific genes by assembling transcriptional activators and repressors to modulate transcription initiation or posttranscriptional regulation [Yoon et al., 2012; Abdelmohsen et al., 2014; Bergmann and Spector, 2014]. Furthermore, LncRNAs may influence cell cycle progression and cell differentiation in different ways, including disturbances of proteostasis, regulation of transcription factors (TFs), and epigenetic alterations [Cesana et al., 2011; Grammatikakis et al., 2014].

In the present study, we evaluated the proliferation and differentiation potential of DPSCs isolated from donors of different ages. In addition, we compared the ex- 
pression profiles of LncRNA and mRNA between DPSCs from young and old donors by LncRNA microarray assays and a bioinformatic analysis. These analyses may help elucidate the senescence mechanism of DPSCs, which will contribute to identifying the key factors necessary to rejuvenate DPSCs from old donors and promoting DPSC-mediated tooth regeneration.

\section{Materials and Methods}

\section{MSCs Isolation and Culture}

Human dental pulps were extracted from healthy teeth without caries, inflammation, or periodontitis that had been extracted due to impacted tooth or orthodontic reasons. The pulps were classified into two age groups (5 teeth per group): a young group (aged 12-25 years) and an old group (aged 60-70 years). DPSCs were separated from the crown and superior two thirds of the root pulp and digested in a solution of $3 \mathrm{mg} / \mathrm{ml}$ collagenase type I (Sigma, St. Louis, Mo., USA) and $4 \mathrm{mg} / \mathrm{ml}$ dispase (Sigma) for $40 \mathrm{~min}$ at $37^{\circ} \mathrm{C}$. Single-cell suspensions were obtained by passing the cells through a $70-\mu \mathrm{m}$ strainer (Falcon, BD Labware, USA). DPSCs were grown in a humidified $5 \% \mathrm{CO}_{2}$ incubator at $37^{\circ} \mathrm{C}$ in $\mathrm{MEM}$ (Gibco, Grand Island, N.Y., USA) supplemented with 15\% fetal bovine serum (Gibco), $2 \mathrm{mM}$ L-glutamine, $100 \mathrm{U} / \mathrm{ml}$ penicillin, and $100 \mathrm{U} / \mathrm{ml}$ streptomycin (Gibco). The culture media were changed every 3 days. DPSCs were used at passages 3-5 in subsequent experiments.

\section{Characterization of MSCs}

Human DPSCs were characterized by flow cytometry using phycoerythrin-conjugated antibodies. DPSC aliquots $\left(1.0 \times 10^{6}\right.$ cells) were incubated for $1 \mathrm{~h}$ at $4{ }^{\circ} \mathrm{C}$ with monoclonal antibodies specific for CD34, CD45, CD105, CD90, CD146, or HLA-DR (all Biolegend, San Diego, Calif., USA). Expression profiles were analyzed by flow cytometry (Calibur; BD Biosciences).

\section{Cell Growth Curve Assays}

DPSCs were seeded at a density of $5.0 \times 10^{4}$ cells/plate on 60 mm plates. Cells were counted 3, 6, and 9 days after seeding. Cells were counted using an automated cell counter (TC10 TM; Bio-Rad Laboratories, Hercules, Calif., USA). Trypan blue was added to the cell suspension to exclude nonviable cells. The results shown represent the means \pm SEM of three separate experiments.

\section{Calculation of Cell Doubling Times}

To assess the population doubling time, cells were counted at $70-80 \%$ confluency. The population doubling time was calculated using the formula $\mathrm{dt}=\mathrm{t} \times \ln _{2} / \ln \left(\mathrm{C}_{\mathrm{t}} / \mathrm{C}_{0}\right)$, where $\mathrm{dt}$ is the doubling time, $t$ is the time between cell count $C_{t}$ and $C 0$ (in h), $C_{0}$ is the initial cell count, and $C_{t}$ is the cell count after time $t$.

\section{Certified Functional Safety Expert Cell Proliferation Assays}

DPSCs were stained according to the CellTrace ${ }^{\mathrm{TM}}$ certified $^{-}$ functional safety expert (CFSE) cell proliferation kit protocol (Invitrogen, USA) for labeling cells in suspension and were then seeded at a density of $5.0 \times 10^{4}$ cells/plate on 6 -well plates. DPSCs were harvested with $0.25 \%$ trypsin after 6 days of culture and analyzed using a flow cytometer (Calibur; BD Biosciences) with 488-nm excitation and emission filters appropriate for fluorescein. The proliferation index was calculated by Modfit LT.

\section{Alkaline Phosphatase Activity Assays and Alizarin Red} Staining

DPSCs were grown in mineralization-inducing medium consisting of MEM, 15\% fetal bovine serum, 2 mM glutamine, $100 \mathrm{U} /$ $\mathrm{ml}$ penicillin, $100 \mathrm{U} / \mathrm{ml}$ streptomycin, $100 \mu \mathrm{M} \mathrm{L}$-ascorbic acid 2-phosphate (Sigma), $10 \mathrm{~mm} \beta$-glycerophosphate (Sigma), and 10 nM dexamethasone (Sigma). Cells were cultured for 3, 5, or 7 days, and alkaline phosphatase (ALP) activity assays were performed using ALP kits according to the manufacturer's protocol (Sigma) and normalized based on protein concentrations. To detect mineralization, cells were induced for 2 or 3 weeks, fixed with $70 \%$ ethanol, and stained with $2 \%$ alizarin red (Sigma). To quantitatively determine calcium mineral content, alizarin red was destained with $10 \%$ cetylpyridinium chloride in $10 \mathrm{~mm}$ sodium phosphate for $60 \mathrm{~min}$ at room temperature. The concentration was determined by absorbance measurement at $562 \mathrm{~nm}$ on a multiplate reader using a standard calcium curve in the same solution. The final calcium level in each group was normalized to the total protein concentrations prepared from a duplicate plate.

\section{Oil Red O Staining}

Adipogenic differentiation was induced using the StemPro adipogenesis differentiation kit (Invitrogen). DPSCs were grown in adipose-inducing medium for 3 weeks. For oil red $\mathrm{O}$ staining, cells were fixed with $10 \%$ formalin for at least $1 \mathrm{~h}$ at room temperature. Next, the cells were stained with the $60 \%$ oil red $\mathrm{O}$ in isopropanol as a working solution for $10 \mathrm{~min}$. Oil red $\mathrm{O}$ dye was eluted with $100 \%$ isopropanol for $10 \mathrm{~min}$, and the OD was measured at $500 \mathrm{~nm}$ using $100 \%$ isopropanol as a blank. The final OD value in each group was normalized to the total protein concentration prepared from a duplicate plate.

\section{Alcian Blue Stain Assays}

Chondrogenic differentiation was induced using a StemPro chondrogenesis differentiation kit (Invitrogen). DPSCs were grown in chondrogenic medium for 2 weeks. For Alcian blue staining, cells were rinsed once with PBS and fixed with $4 \%$ formaldehyde solution for $30 \mathrm{~min}$. After fixation, the wells were rinsed with PBS, and the cells were stained for 30 min with a $1 \%$ Alcian blue solution prepared in $0.1 \mathrm{~N} \mathrm{HCl}$. Then, the wells were rinsed three times with $0.1 \mathrm{~N} \mathrm{HCl}$, and distilled water was added to neutralize the acidity. Blue staining indicated the synthesis of proteoglycans by chondrocytes. To quantify proteoglycan synthesis, Alcian blue was extracted by $4 \mathrm{M}$ guanidine- $\mathrm{HCl}$ overnight at $4^{\circ} \mathrm{C}$. Absorbance values were read at $600 \mathrm{~nm}$ after temperature equilibration. The final OD value in each group was normalized to the total protein concentrations prepared from a duplicate plate.

\section{RNA Preparation and Microarray Analysis}

DPSCs were grown to $90 \%$ confluence. Then, DPSCs from young donors $(n=4)$ and DPSCs from old donors $(n=4)$ were briefly rinsed with PBS and lysed in TRIzol reagent (Invitrogen) for RNA extraction. Total RNA was quantified using a NanoDrop 2000 (Thermo Scientific), and RNA integrity was assessed using an Agilent Bioanalyzer 2100 (Agilent Technologies). The sample labeling, microarray hybridization, and washing were performed 
based on the manufacturer's standard protocols. Briefly, total RNA was transcribed to double-strand cDNA and was then synthesized into cRNA and labeled with cyanine-3-CTP. The labeled cRNAs were hybridized onto the microarray. After washing, the arrays were scanned using an Agilent Scanner G2505C (Agilent Technologies).

\section{Reverse Transcription Polymerase Chain Reaction}

Total RNA was isolated from the MSCs using TRIzol reagents (Invitrogen). We synthesized cDNA from 500-ng aliquots of total RNA with random primers and reverse transcriptase according to the manufacturer's protocol. We performed real-time polymerase chain reaction (PCR) using QuantiTect SYBR Green PCR kits (Qiagen, Hilden, Germany) and an iCycler iQ multicolor real-time PCR detection system (Bio-Rad, Hercules, Calif., USA). Changes in gene expressions were evaluated by normalizing the PCR signal to that of GAPDH and applying the $2^{-\Delta \Delta \mathrm{C}_{\mathrm{T}}}$ calculation. Each reaction was run in triplicate, and the entire procedure was repeated three times. The primers are listed in online supplementary table 1 (see www.karger.com/doi/10.1159/000448026 for all online suppl. material).

\section{Bioinformatic Analysis and Statistics}

Feature extraction software (version 10.7.1.1, Agilent Technologies) was used to analyze the array images to obtain raw data. GeneSpring software was employed to finish the basic analysis with the raw data. First, the raw data were normalized using the quantile algorithm. The probes for which at least 1 of 2 conditions had $75 \%$ flags in 'P' were chosen for further data analyses. Differentially expressed genes or LncRNAs were then identified based on fold changes and p values calculated using t tests. The threshold set for up- and down-regulated genes was a fold change $\geq 2.0$ and a value of $\mathrm{p} \leq 0.05$. Afterwards, gene ontology (GO) analyses and Kyoto Encyclopedia of Genes and Genomes (KEGG) analyses were applied to determine the roles of these differentially expressed mRNAs. Finally, hierarchical clustering was performed to display the distinguishable gene expression patterns among the samples.

\section{Statistics}

All statistical calculations were performed using SPSS 10 statistical software. Student's t test was performed to determine statistical significance. A value of $\mathrm{p} \leq 0.05$ was considered significant.

\section{Results}

DPSCs Isolated from Old Donors Exhibited Decreased Cell Proliferation Ability

According to the flow cytometry analysis, these two groups were both negative for cell surface markers CD34, CD45, and HLA-DR, and positive for CD90, CD105, and CD146 (online suppl. fig. 1). There were no significant differences between young and old groups.

Cell proliferation was monitored over a period of 9 days, and we found that DPSCs from young donors exhibited enhanced proliferation ability compared to
DPSCs from old donors (fig. 1a). Moreover, the average doubling times were significantly different between DPSCs from young donors $(28.54 \pm 1.83 \mathrm{~h})$ and DPSCs from old donors $(46.74 \pm 6.37 \mathrm{~h})(\mathrm{p}<0.05)$. To further confirm the proliferation results, we detected the cell proliferation indexes by CFSE cell proliferation assays. The result also showed that the cell indexes of DPSCs from young donors were much higher than those of DPSCs from old donors (fig. 1b-d).

\section{DPSCs Isolated from Old Donors Exhibited Impaired}

Osteogenic and Adipogenic Differentiation Potentials

The osteogenic differentiation potentials were investigated by culturing DPSCs with osteogenic-inducing medium. The ALP activity, an early maker of osteogenic differentiation, was higher in the DPSCs from young donors than in the DPSCs from old donors 7 days after induction (fig. 1e). After culturing DPSCs in osteogenic-inducing medium for 2 and 3 weeks, alizarin red staining and calcium quantitation revealed that mineralization was also significantly stronger in the DPSCs from young donors than in the DPSCs from old donors (fig. 1f, g). We further investigated various osteogenic differentiation makers, including osteopontin (OPN), osteocalcin, collagen (COL) type I $\alpha_{2}$ (COL1A2), and the key TF runt-related TF 2 (RUNX2) by real-time PCR. The results showed that OPN expression was increased 1 and 3 weeks after osteogenic induction in DPSCs from young donors compared to DPSCs from old donors (fig. 1h). However, the expression levels of COL1A2, RUNX2, and osteocalcin were not significantly different between these two groups (data not shown).

We also compared the adipogenic differentiation potentials of DPSCs from different donors. After induction with adipogenic medium for 3 weeks, the oil red staining and a quantitation analysis revealed that DPSCs from young donors had more lipid deposits than DPSCs from old donors after induction (fig. 2a, b). We further examined the adipogenic differentiation marker, lipoprotein lipase (LPL), and CCAAT/enhancer binding protein a (CEBPA) by real-time PCR. The result showed that the expression of LPL was higher 2 and 3 weeks after adipogenic induction in DPSCs from young donors than in DPSCs from old donors (fig. 2c). The expression of CEBPA was not significantly different between these two groups (data not shown).

In addition, we compared the chondrogenic differentiation potential of DPSCs from young and old groups. After induction with chondrogenic medium for 2 weeks, Alcian blue staining and a quantitation analysis revealed 


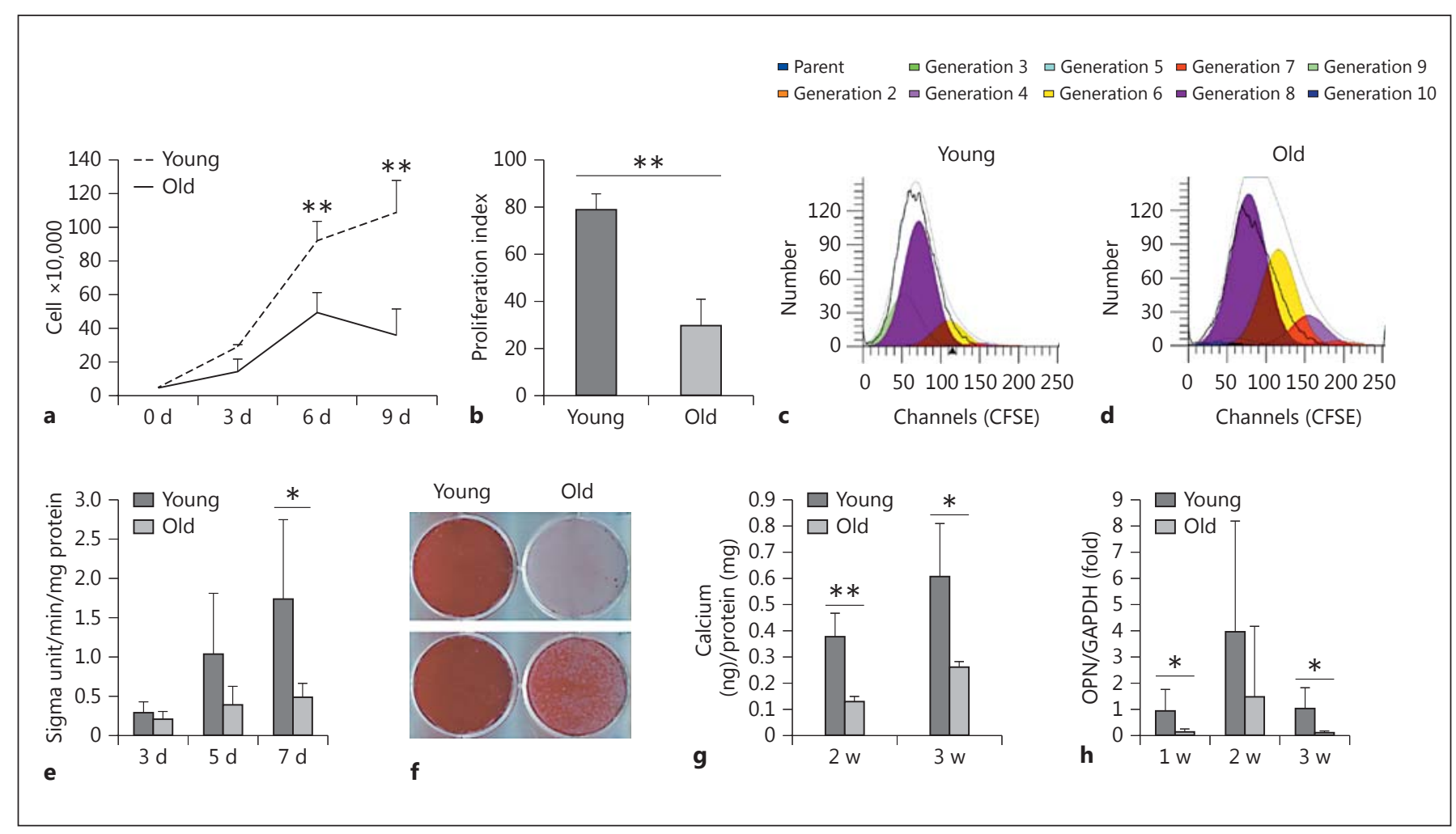

Fig. 1. The cell proliferation capacity and osteogenic potential were decreased in DPSCs from old donors compared with DPSCs from young donors. a Cell growth curves showed that DPSCs from young donors grew faster than DPSCs from old donors $(\mathrm{p}<0.05)$. b-d CFSE cell proliferation assays showed that the cell indexes of DPSCs from young donors were much higher than those of DPSCs from old donors. e ALP activity was enhanced in DPSCs from young donors compared with DPSCs from old donors after osteo- genic induction. $\mathbf{f}, \mathbf{g}$ Alizarin red staining ( $\mathbf{f}$ ) and calcium quantitation (g) showed that mineralization was also significantly higher in DPSCs from young donors than in DPSCs from old donors. h Real-time PCR results indicated the expression of OPN in DPSCs from young and old donors. GAPDH was used as an internal control. Student's t tests were performed to determine statistical significance $\left({ }^{*} \mathrm{p}<0.05,{ }^{* *} \mathrm{p}<0.01\right)$. Error bars $=\mathrm{SD}(\mathrm{n}=5)$; $\mathrm{d}=$ days; $\mathrm{w}=\operatorname{week}(\mathrm{s})$. similar amounts of proteoglycan production in the DPSCs from young and old donors (fig. 2d, e). Real-time PCR showed that the levels of the chondrogenic differentiation markers COL2, SOX9, and COL5 were not significantly different between the groups (fig. $2 \mathrm{f}-\mathrm{h}$ ).

\section{Comparison of LncRNA and mRNA Expression}

Profiles between DPSCs from Young and Old Donors

To illuminate why DPSCs from different age groups showed different proliferation and differentiation potentials, we screened the LncRNA and mRNA expression patterns of DPSCs from young and old donors using the human LncRNA GeneChip. From the microarray data, a comparison of the LncRNA expression levels identified a total of 561 LncRNAs that were significantly differentially expressed, of which 389 were up-regulated and 172 were down-regulated in the DPSCs from young donors compared to the DPSCs from old donors (online suppl. table 2). In addition, a total of 304 mRNAs were differentially expressed between the young and old groups, of which 247 were up-regulated and 57 were downregulated in the DPSCs from young donors compared to the DPSCs from old donors (online suppl. table 3). To confirm the reliability of the microarray data, 4 differentially expressed LncRNAs (NONHSAT003823, NONHSAT007265, NR_037840.2, and NR_024047.1) and 6 differentially expressed mRNAs [G-proteincoupled receptor class C group 5 member A (GPRC5A), F-box protein 5 (FBXO5), wingless-type MMTV integration site family (WNT) 2, centromere protein $\mathrm{K}$ (CENPK), abnormal spindle microtubule assembly (ASPM), and peroxisome proliferator-activated receptor- $\gamma$ (PPARG) were selected, and real-time PCR was performed to detect their expression in DPSCs from the 


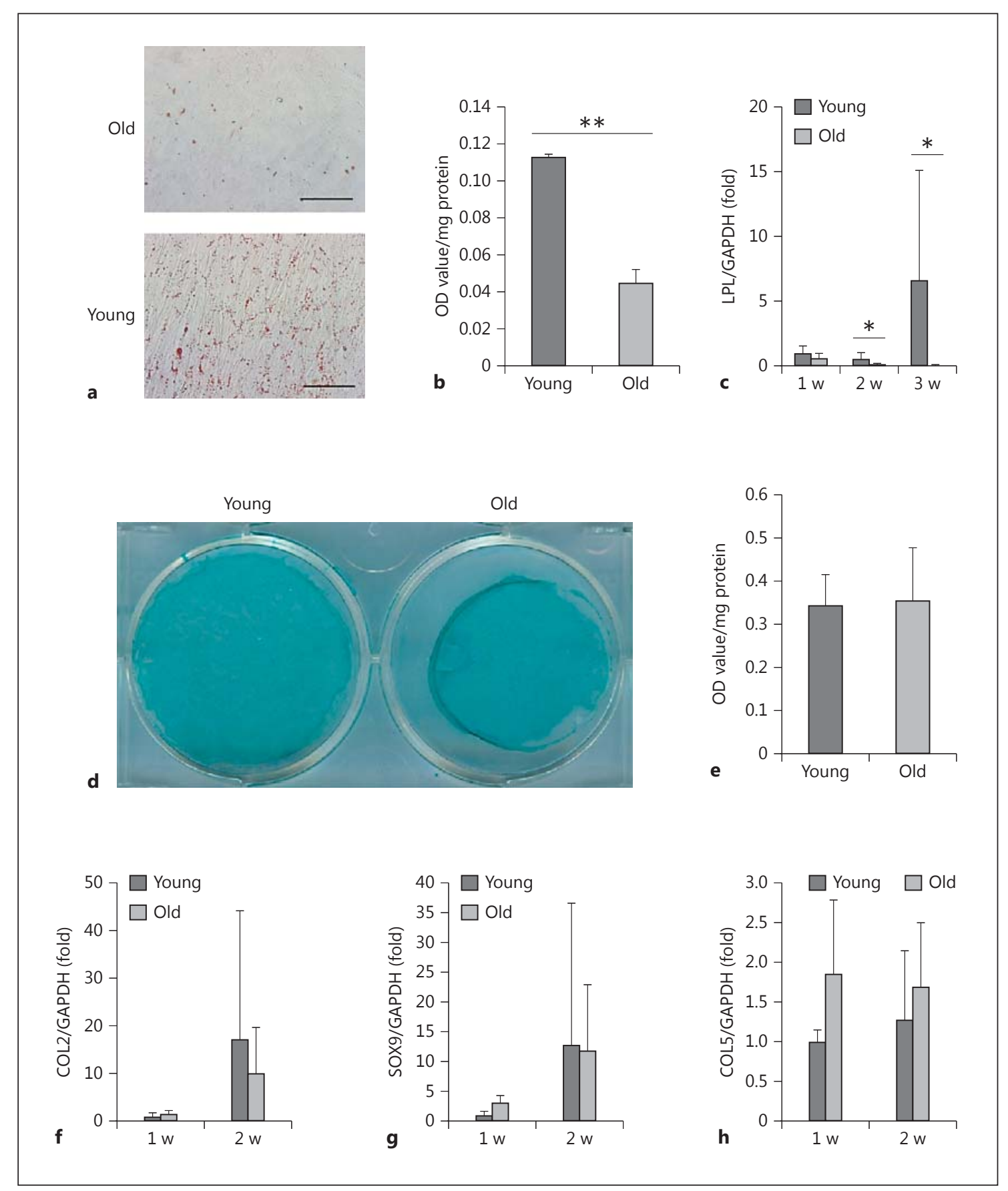

Fig. 2. The adipogenic differentiation potentials, but not chondrogenic differentiation potentials, were decreased in DPSCs from old donors compared with DPSCs from young donors. a Oil red staining revealed that DPSCs from young donors had more lipid deposits than DPSCs from old donors. Bar $=100 \mu \mathrm{m}$. b Quantification of lipid droplet confirmed that adipogenic potential was decreased in DPSCs from old donors compared with DPSCs from young donors. c Real-time PCR results showed that the expression of LPL was higher in DPSCs from young donors than in DPSCs from old donors after adipogenic induction. $\mathbf{d}$ Alcian blue staining revealed similar amounts of proteoglycan production in DPSCs from young and old donors. e Quantification of proteoglycan synthesis at 2 weeks. f-h Real-time PCR results showed the expression of COL2, SRY (sex-determining region Y)-box 9 (SOX9), and COL5 after chondrogenic induction. GAPDH was used as an internal control. Student's t tests were performed to determine statistical significance $\left({ }^{*} \mathrm{p}<0.05,{ }^{* *} \mathrm{p}<0.01\right)$. Error bars $=\mathrm{SD}(\mathrm{n}=5) ; \mathrm{w}=\operatorname{week}(\mathrm{s})$. 


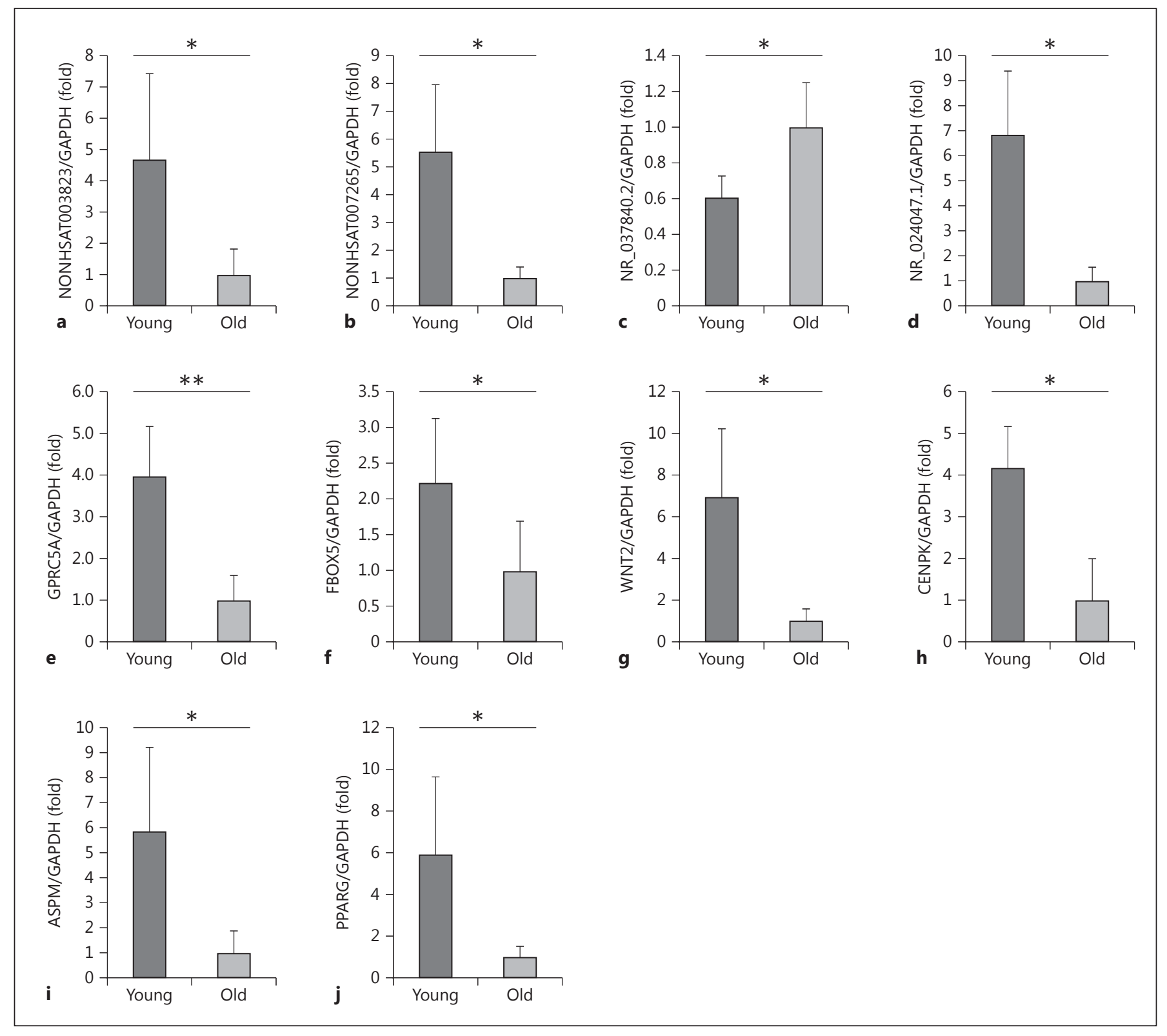

Fig. 3. Gene expression detected by real-time PCR in DPSCs from young and old donors. GAPDH was used as internal control. Student's t tests were performed to determine statistical significance $\left({ }^{*} \mathrm{p}<0.05,{ }^{* *} \mathrm{p}<0.01\right)$. Error bars $=\mathrm{SD}(\mathrm{n}=5)$.

young and old donors. The expression levels of the 4 selected LncRNAs and the 6 selected mRNAs were consistent with the microarray results and confirmed the reliability of the microarray data (fig. 3 ).

\section{Bioinformatic Analysis of the Microarray Data}

Finally, a bioinformatic analysis was performed to discover the key factors that control the DPSC aging process.
We identified co-expressed mRNAs of LncRNAs by calculating the Pearson correlations and then chose the significant correlation pairs to calculate the enrichment of functional terms in annotation of co-expressed mRNAs. First, a functional analysis was executed to predict the main function of the differentially expressed LncRNAs according to the $\mathrm{GO}$ analysis, which is the key functional classification of the National Center for Biotechnology 


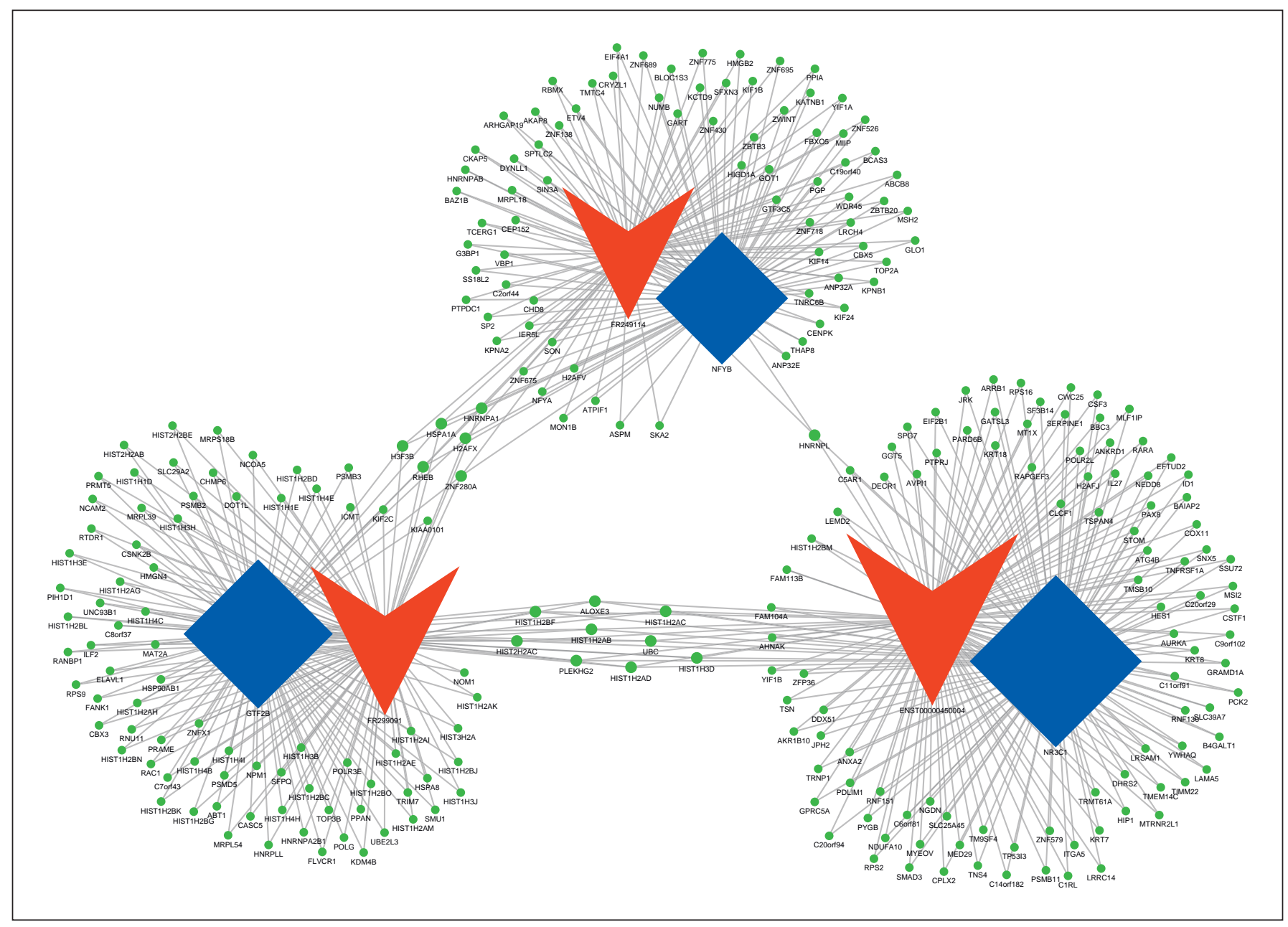

Fig. 4. The ternary relation network diagram according to the top 500 LncRNA-gene correlation pairs and TFgene correlation pairs. The diagram indicates that NFYB, GTF2B, and NR3C1 may be core TFs and that FR249114, FR299091, and ENST00000450004 may be core LncRNAs in the senescence of DPSCs. The blue nodes represent TFs, the red nodes represent LncRNAs, and the green nodes represent the target genes.

Information. The result showed that the top 5 GOs were responsible for chromatin binding, nucleosomal DNA binding, DNA polymerase binding, protein heterodimerization activity, and single-stranded DNA binding (online suppl. fig. 2). Similarly, a pathway analysis was applied to determine the significant pathway of the differentially expressed LncRNAs and mRNAs according to KEGG. The result revealed the viral carcinogenesis, cell cycle, RNA transport, the mRNA surveillance, and the Fanconi anemia pathways were the top 5 functional pathways (online suppl. fig. 3). We also used a hypergeometric cumulative distribution function to calculate the enrichment of TFs in the annotation of co-expressed mRNAs, and we selected the top 200 LncRNA-TF correlation pairs according to the q-value to calculate the functional annotation frequencies of these TFs. We found that the top 5 TFs were interferon regulatory factor 3 (IRF3), general TF IIF subunit 1 (GTF2F1), SIN3 transcription regulator family member A, TATA-box binding protein (TBP), and general TF IIB (GTF2B) (online suppl. fig. 4).

To further investigate the global network, we used the result of the hypergeometric cumulative distribution analysis to draw a visual network diagram. The binary relation network diagram of the top 100 LncRNA-TF correlation pairs showed that the IRF3, TBP, GTF2F1, and nuclear TF Y (NFY) subunit a (NFYA) TFs were impor- 
tant according to the high-betweenness centrality calculation. Among these TFs, TBP and IRF3 were the key TFs with the best connectivities and interactions with 9 LncRNAs, and TBP was the node in the single net with the most powerful mediation ability (online suppl. fig. 5). Finally, we constructed a ternary relation network diagram according to the top 500 LncRNA-gene correlation pairs and TF-gene correlation pairs to evaluate the interactions among genes, LncRNAs, and TFs and to locate the core regulatory LncRNAs and TFs in the network. The diagram describes the interaction among TFs, LncRNAs, and target genes. NFY subunit $\beta$ (NFYB), GTF2B, and nuclear receptor subfamily 3 group $\mathrm{C}$ member 1 (NR3C1) were identified as the core TFs, and FR249114, FR299091, and ENST00000450004 were identified as the core LncRNAs (fig. 4).

\section{Discussion}

The dental pulp tissue, similar to other tissues, undergoes age-depended changes that may lead to the loss of vitality of dentin pulp [Domine and Holz, 1991]. Although many studies have investigated the mechanisms underlying these age-related changes, there is still much to learn on the biological mechanisms responsible for cellular vitality and survival throughout pulp life.

Stem cells play an important role in maintaining the biological function of tissue. In this study, we used DPSCs isolated from donors of different ages and investigated the age-related characteristics and expression profiles of the DPSCs. First, the results revealed that DPSCs from young donors possessed a stronger proliferation ability compared with DPSCs from old donors, which is consistent with age-related changes that occur in other types of MSCs [Kretlow et al., 2008; Zaim et al., 2012; Zhang et al., 2012; Choudhery et al., 2014]. Next, impaired osteogenic and adipogenic differentiation potential was found in DPSCs from old donors compared with DPSCs from young donors. However, Alcian blue staining and a chondrogenic differentiation marker analysis indicated that there was no difference in the chondrogenic differentiation potential of DPSCs from donors with different ages. These results suggest that DPSCs from old donors are not as good as DPSCs from young donors for cell proliferation and differentiation, which is in accordance with the results of a recent investigation [Horibe et al., 2014]. This investigation also discovered that mobilized DPSCs (MDPSCs), which are DPSC subsets based on their migratory response to granulocyte-colony stimulating fac-

Effect of Age on DPSC Characteristics and Gene Expression tor, from aged donors are similar to young donors in their proliferation capacity, regeneration potential, migration, and differentiation [Horibe et al., 2014]. These results suggest that the biological characteristics of DPSCs may be affected by different isolation methods. We investigated the biological characteristics of DPSCs isolated by the traditional method, which is more commonly used, and found that they are influenced by donor age. Some researchers have reported that in vitro assays that measure MSC proliferation and viability can be used to predict the in vivo therapeutic ability of MSCs [Deskins et al., 2013]. MSCs with more powerful differentiation potentials will be beneficial for special tissue regeneration. Our in vitro discoveries strongly suggest that these senescence-related impaired functions of DPSCs would influence in vivo pulp tissue vitality and pulp regeneration.

To clarify the mechanism of the aging process in DPSCs, we performed an LncRNA microarray analysis. We screened the differentially expressed mRNAs and LncRNAs of DPSCs from young and old donors. According to the GeneChip analysis, 561 LncRNAs were identified to be differentially expressed, including 389 up-regulated and 172 down-regulated LncRNAs in the DPSCs from young donors compared to the DPSCs from old donors. In addition, a total of 304 mRNAs were differentially expressed in the young and old groups, of which 247 were up-regulated and 57 were down-regulated in the young group compared to the old group. Furthermore, we found that several of the differentially expressed genes in the two groups may be associated with age-related declining properties of DPSCs. For example, PPARG was reported as a regulator of adipocyte differentiation implicated in the pathology of obesity [Janesick and Blumberg, 2011]. In addition, WNT2 and WNT2B are both signaling pathways that play important roles in the differentiation and proliferation of cementoblasts and odontoblasts and in the formation of teeth [Tamura et al., 2010].

Furthermore, we applied a bioinformatics analysis to classify the microarray results. The GO analysis revealed that most of the differentially expressed LncRNAs and mRNAs were functionally associated with cell proliferation. In addition, the KEGG analysis also showed that the functional pathways that were enriched in the genes were primarily associated with cell and tissue proliferation, differentiation, and DNA repair. These results were in agreement with a previous study that compared the gene expression profiles of human dental pulp in young and older subjects by a microarray analysis [Tranasi et al., 2009].

To identify important TFs involved in the senescence of DPSCs, we executed a correlation analysis to compare 
LncRNAs and TFs using a hypergeometric cumulative distribution function. The analysis revealed that IRF3 exhibited the highest functional annotations. Moreover, a binary relation network diagram of the top 100 LncRNATF correlation pairs clarified the relationship between LncRNAs and TFs. The result showed that IRF3 and TBP were the key TFs with the best connectivities and interactions with 9 LncRNA. In addition, TBP was the key node in the single net with the most powerful mediation ability. It has been reported as required for transcription by all three classes of nuclear RNA polymerases found in eukaryotes [White and Jackson, 1992]. IRF3 activates the transcription of interferon- $\alpha$ and $-\beta$ and other interferon-induced genes [Au et al., 1995; Lin et al., 2014]. Finally, a ternary relation network diagram revealed that NFYB, GTF2B, and NR3C1 were the core TFs, and FR249114, FR299091, and ENST00000450004 were the core LncRNAs. NFYB, a subunit of NFY that binds to the CCAAT box, is a sequence-specific TF with nucleosomelike properties of nonspecific DNA binding and helps establish permissive chromatin modifications at CCAAT promoters [Nardini et al., 2013] and could interact with TBP [Bellorini et al., 1997]. GTF2B is one of the ubiquitous factors required for transcription initiation by RNA polymerase II and was reported to have a potential link to cell proliferation and growth [Li et al., 2013]. NR3C1, a glucocorticoid receptor, is involved in cellular proliferation and differentiation in target tissues [Wintermantel et al., 2005; Rauch et al., 2011; Ertaylan et al., 2014]. Our results are in accordance with these previous reports and may also be applied to the biological mechanisms of the senescence of DPSCs. LncRNAs may exert their function by controlling the transcriptional regulation of neighboring coding genes [Hung and Chang, 2010; Ma et al., 2012]. Moreover, we also identified various differentially expressed LncRNAs in age-related DPSCs and nearby differentially expressed mRNAs, which may be associated with or modulated by these LncRNAs and will aid in the study of LncRNA function. Further studies should be conducted to determine their roles and biological characteristics in the senescence of DPSCs.

In summary, our study demonstrated that the proliferation and osteogenic and adipogenic potentials of DPSCs from old donors were decreased compared to DPSCs from young donors, indicating that senescence impaired the proliferation and differentiation potentials of DPSCs and that donor age is an important factor that affects their use in tissue regeneration. In addition, these proliferation and differentiation property differences among age-related DPSCs may be associated with the age-related changes in dental pulp. Our study also provides comprehensive mRNA and LncRNA profiles of DPSCs from young and old donors. Potential regulatory mechanisms were identified by a bioinformatic analysis. Although more studies are required to demonstrate the precise role and mechanisms of these LncRNAs and mRNAs, the genomic data we identified based on the microarray analyses may increase our understanding of the senescence characteristics and mechanisms of MSCs.

\section{Acknowledgment}

This work was supported by grants from the National Natural Science Foundation of China (81271101 to Z.F. and 81300841 to O.L), the Program for New Century Excellent Talents in University (NCET-12-0611 to Z.F.), the High-level Talents of the Beijing Health System (2013-3-035 to Z.F.), the Program for 'HundredThousand-Ten thousand' Talents in Beijing (2014006 to Z.F.), the Chinese Medicine Science and Technology Foundation of Beijing (JJ2015-10 to Z.F.), the Science and Technology Department of Hunan Province (2014FJ6031 to Y.L.), and the Fundamental Research Funds for the Central Universities of Central South University (2014zzts306 to Q.Y.).

\section{Disclosure Statement}

The authors have no potential conflicts of interest.

\section{References}

Abdelmohsen, K., A.C. Panda, M.J. Kang, R. Guo, J. Kim, I. Grammatikakis, J.H. Yoon, D.B. Dudekula, J.H. Noh, X. Yang, J.L. Martindale, M. Gorospe (2014) 7SL RNA represses p53 translation by competing with HuR. Nucleic Acids Res 42: 10099-10111.

$\mathrm{Au}$, W.C., P.A. Moore, W. Lowther, Y.T. Juang, P.M. Pitha (1995) Identification of a member of the interferon regulatory factor family that binds to the interferon-stimulated response element and activates expression of interferon-induced genes. Proc Natl Acad Sci USA 92: 11657-11661.

Bellorini, M., D.K. Lee, J.C. Dantonel, K. Zemzoumi, R.G. Roeder, L. Tora, R. Mantovani (1997) CCAAT binding NF-Y-TBP interactions: NF-YB and NF-YC require short domains adjacent to their histone fold motifs for association with TBP basic residues. Nucleic Acids Res 25: 2174-2181.
Bergmann, J.H., D.L. Spector (2014) Long noncoding RNAs: modulators of nuclear structure and function. Curr Opin Cell Biol 26: $10-18$.

Cesana, M., D. Cacchiarelli, I. Legnini, T. Santini, O. Sthandier, M. Chinappi, A. Tramontano, I. Bozzoni (2011) A long noncoding RNA controls muscle differentiation by functioning as a competing endogenous RNA. Cell 147: 358369 .
10

Cells Tissues Organs 2017;203:1-11

DOI: $10.1159 / 000448026$
Yi/Liu/Yan/Lin/Diao/Wang/Jin/Wang/ $\mathrm{Lu} /$ Fan 
Choudhery, M.S., M. Badowski, A. Muise, J. Pierce, D.T. Harris (2014) Donor age negatively impacts adipose tissue-derived mesenchymal stem cell expansion and differentiation. J Transl Med 12: 8.

Deskins, D.L., D. Bastakoty, S. Saraswati, A. Shinar, G.E. Holt, P.P. Young (2013) Human mesenchymal stromal cells: identifying assays to predict potency for therapeutic selection. Stem Cells Transl Med 2: 151-158.

Domine, L., J. Holz (1991) The aging of the human pulp-dentin organ (in French). Schweiz Monatsschr Zahnmed 101: 725-733.

Ertaylan, G., S. Okawa, J.C. Schwamborn, S.A. Del (2014) Gene regulatory network analysis reveals differences in site-specific cell fate determination in mammalian brain. Front Cell Neurosci 8: 437.

Grammatikakis, I., A.C. Panda, K. Abdelmohsen, M. Gorospe (2014) Long noncoding RNAs (LncRNAs) and the molecular hallmarks of aging. Aging 6: 992-1009.

Horibe, H., M. Murakami, K. Iohara, Y. Hayashi, N. Takeuchi, Y. Takei, K. Kurita, M. Nakashima (2014) Isolation of a stable subpopulation of mobilized dental pulp stem cells (MDPSCs) with high proliferation, migration, and regeneration potential is independent of age. PLoS One 9: e98553.

Hung, T., H.Y. Chang (2010) Long noncoding RNA in genome regulation: prospects and mechanisms. RNA Biol 7: 582-585.

Iohara, K., M. Murakami, N. Takeuchi, Y. Osako, M. Ito, R. Ishizaka, S. Utunomiya, H. Nakamura, K. Matsushita, M. Nakashima (2013) A novel combinatorial therapy with pulp stem cells and granulocyte colony-stimulating factor for total pulp regeneration. Stem Cells Transl Med 2: 521-533.

Janesick, A., B. Blumberg (2011) Minireview: PPAR $\gamma$ as the target of obesogens. J Steroid Biochem Mol Biol 127: 4-8.
Kornicka, K., K. Marycz, K.A. Tomaszewski, M. Marędziak, A. Śmieszek (2015) The effect of age on osteogenic and adipogenic differentiation potential of human adipose derived stromal stem cells (hASCs) and the impact of stress factors in the course of the differentiation process. Oxid Med Cell Longev 2015: 309169.

Kretlow, J.D., Y.Q. Jin, W. Liu, W.J. Zhang, T.H. Hong, G. Zhou, L.S. Baggett, A.G. Mikos, Y. Cao (2008) Donor age and cell passage affects differentiation potential of murine bone marrow-derived stem cells. BMC Cell Biol 9: 60.

Li, L., A. Zhang, X. Cao, J. Chen, Y. Xia, H. Zhao, A. Shen (2013) General transcription factor IIb overexpression and a potential link to proliferation in human hepatocellular carcinoma. Pathol Oncol Res 19: 195-203.

Lin, L., S. Pan, J. Zhao, C. Liu, P. Wang, L. Fu, X. $\mathrm{Xu}, \mathrm{M}$. Jin, A. Zhang (2014) HSPD1 interacts with IRF3 to facilitate interferon-beta induction. PLoS One 9: e114874.

López-Otín, C., M.A. Blasco, L. Partridge, M. Serrano, G. Kroemer (2013) The hallmarks of aging. Cell 153: 1194-1217.

Ma, H., Y. Hao, X. Dong, Q. Gong, J. Chen, J. Zhang, W. Tian (2012) Molecular mechanisms and function prediction of long noncoding RNA. ScientificWorldJournal 2012: 541786.

Nardini, M., N. Gnesutta, G. Donati, R. Gatta, C. Forni, A. Fossati, C. Vonrhein, D. Moras, C. Romier, M. Bolognesi, R. Mantovani (2013) Sequence-specific transcription factor NF-Y displays histone-like DNA binding and H2Blike ubiquitination. Cell 152: 132-143.

Rauch, A., V. Gossye, D. Bracke, E. Gevaert, P. Jacques, K. Van Beneden, B. Vandooren, M. Rauner, L.C. Hofbauer, G. Haegeman, D. Elewaut, J.P. Tuckermann, K. De Bosscher (2011) An anti-inflammatory selective glucocorticoid receptor modulator preserves osteoblast differentiation. FASEB J 25: 1323-1332.

Tamura, M., E. Nemoto, M.M. Sato, A. Nakashima, H. Shimauchi (2010) Role of the Wnt signaling pathway in bone and tooth. Front Biosci (Elite Ed) 2: 1405-1413.
Tranasi, M., M.T. Sberna, V.Zizzari, G. D’Apolito, F. Mastrangelo, L. Salini, L. Stuppia, S. Tete (2009) Microarray evaluation of age-related changes in human dental pulp. J Endod 35: 1211-1217.

Wei, F., T. Song, G. Ding, J. Xu, Y. Liu, D. Liu, Z. Fan, C. Zhang, S. Shi, S. Wang (2013) Functional tooth restoration by allogeneic mesenchymal stem cell-based bio-root regeneration in swine. Stem Cells Dev 22: 1752-1762.

White, R.J., S.P. Jackson (1992) The TATA-binding protein: a central role in transcription by RNA polymerases I, II and III. Trends Genet 8: $284-288$

Wintermantel, T.M., D. Bock, V. Fleig, E.F. Greiner, G. Schutz (2005) The epithelial glucocorticoid receptor is required for the normal timing of cell proliferation during mammary lobuloalveolar development but is dispensable for milk production. Mol Endocrinol 19: 340-349.

Yen, A.H., P.C. Yelick (2011) Dental tissue regeneration - a mini-review. Gerontology 57: $85-$ 94.

Yoon, J.H., K. Abdelmohsen, S. Srikantan, X. Yang, J.L. Martindale, S. De, M. Huarte, M. Zhan, K.G. Becker, M. Gorospe (2012) LincRNA-p21 suppresses target mRNA translation. Mol Cell 47: 648-655.

Yu, J., Y. Wang, Z. Deng, L. Tang, Y. Li, J. Shi, Y. Jin (2007) Odontogenic capability: bone marrow stromal stem cells versus dental pulp stem cells. Biol Cell 99: 465-474.

Zaim, M., S. Karaman, G. Cetin, S. Isik (2012) Donor age and long-term culture affect differentiation and proliferation of human bone marrow mesenchymal stem cells. Ann Hematol 91: 1175-1186.

Zhang, J., Y. An, L.N. Gao, Y.J. Zhang, Y. Jin, F.M. Chen (2012) The effect of aging on the pluripotential capacity and regenerative potential of human periodontal ligament stem cells. Biomaterials 33: 6974-6986. 